International Journal of Engineering \& Technology, $7(4.10)(2018) 570-572$
SPC
International Journal of Engineering \& Technology
Website: www.sciencepubco.com/index.php/IJET
Research paper

\title{
Some Results on Generating Graceful Trees
}

\author{
S.Venkatesh $^{1, *}$, K. Balasubramanian ${ }^{2}$ \\ ${ }^{1,2}$ SASTRA deemed UNIVERSITY, SRC Campus, Kumbakonam - 612 001, Tamilnadu, India \\ *Corresponding author, E-mail:mailvenkat1973@gmail.com,${ }^{2} k \_$bala27@yahoo.co.in
}

\begin{abstract}
Let $T$ and $S$ be any two simple graphs. Then $T+S$ is the graph obtained by merging a vertex of each copy of $S$ with every attachment vertices of $T$. Let $T_{0}$ be the one vertex union of copies of the given caterpillar $T$ with the common vertex as one of the penultimate vertices. If $S_{0}$ is any caterpillar, then define $T_{1}=T_{0}+S_{0}$. Recursively for $i \geq 2$, construct $T_{i}$, that is, $T_{i}=T_{i-1}+S_{i-1}$. Here the tree $S_{i-1}$ considered for attachment with $T_{i-1}$ is a caterpillar, but not necessarily the same among the levels. In this paper we prove that the tree $T_{i}$ is graceful for $i \geq 1$.
\end{abstract}

Keywords: Trees; Graceful labeling; Recursive attachment.

\section{Introduction}

In 1963, Ringel conjectured that $T$ is a tree with $m$ edges, then the complete graph $K_{2 m+1}$ can be decomposed into $2 m+1$ subgraphs isomorphic to $T$. Kotzig has given the strengthened form of this conjecture and in 1967, Rosa(see [2]) introduced various ways of numbering the vertices of $T$ as a tool to attack the Ringel-Kotzig conjecture. In 1968, the word graceful is coined by Golomb (see $[1])$ and it is the term widely used. A graceful labeling of a graph $G(V, E)$ with $m$ edges is an injection $f: V(G) \rightarrow\{0,1,2, \ldots, m\}$ with the property that resulting edge labels are distinct, where an edge $u v$ is assigned the label $|f(u)-f(v)|$. A graph which admits graceful labeling is called a graceful graph.

There are many graphs shown to be graceful. Some of the known family of trees are caterpillars, symmetrical trees(see [5]),[10]), banana trees(see [11], [12]), spiders(see [4]). Further, Stanton and Zarnke (see [3]) Koh, Rogers and Tan(see [9] provided methods for combining graceful trees to generate larger graceful trees from the known graceful trees. Burzio and Ferrarese(see [6]) have shown that subdivision of a graceful tree is graceful. Sethuraman and Jesintha (see [13]) have provided a method to generate graceful lobster from a graceful caterpillar. Sethuraman and Ragukumar (see [14]) provided an algorithm that generates a graceful tree from a given arbitrary tree by adding a sequence of new pendent edges to the given arbitrary tree thereby proving that every tree is a subtree of a graceful tree. A complete survey on graceful labeling is done by Gallian (see [8]). Graceful graphs have wide applications in coding theory, X-ray crystallography, circuit design, radio communication, networks and radio astronomy.

Definition 1.1: A vertex $u$ is said to be an attachment vertex if $\operatorname{deg}(u) \geq 2$.

Definition 1.2: A vertex of a caterpillar which has at most one adjacent vertex of degree greater than or equal to two is called a penultimate vertex and represent it as $v_{f}$ and $v_{l}$.
Let $T$ and $S$ be any two simple graphs. Then $T+S$ is the graph obtained by merging the penultimate vertex (fixed) of each copy of $S$ with every attachment vertices of $T$. Let $T_{0}$ be the one vertex union of copies of the given caterpillar $T$ with the common vertex as one of the penultimate vertices. If $S_{0}$ is any caterpillar, then define $T_{1}=T_{0}+S_{0}$. For $i \geq 2$, define $T_{i}=T_{i-1}+S_{i-1}$ recursively by attaching each copy of $S_{i-1}$ with every vertices of degree greater or equal to two of $T_{i-1}$. Here the tree considered for attachment with $T_{i}$ is a caterpillar, but not necessarily the same among the levels. In this paper, we prove that the tree $T_{i}$ is graceful for $i \geq 1$. In the next section let us first prove that the tree $T_{1}$ is graceful.

\section{2. $T_{1}$ is graceful}

Let $T$ be any caterpillar with the penultimate vertices $v_{f}$ and $v_{l}$. Consider $r$ copies of $T$ and let it be $T^{1}, T^{2}, T^{3}, \ldots T^{r}$. Then $T_{0}$ is the one vertex union of $T^{i}$, for $1 \leq i \leq r$ with the common vertex as $v_{f}$, having $t$ attachment vertices. Let $S_{0}$ be any caterpillar and $S_{0}^{1}, S_{0}^{2}, S_{0}^{3}, \ldots, S_{0}^{t}$ be the copies of $S_{0}$. Now the tree $T_{1}\left(=T_{0}+S_{0}\right)$ is constructed by merging the penultimate vertex of each copy of $S_{0}^{j}$ with every attachment vertex of $T_{0}$. With this construction, in the next theorem we prove that $T_{1}$ is graceful.

Theorem 2.1: The tree $T_{1}$ is graceful.

Proof: Let $m$ be the number of edges of $T_{1}$. Now for $1 \leq i \leq r$ name the unlabelled attachment vertices of each copy $S_{0}^{i}$ in the direction from $v_{l}$ to $v_{f}$ or $v_{f}$ to $v_{l}$ as $i$ is odd or even and let it be $u_{1}, u_{2}, u_{3}, \ldots u_{s}$.

Let $P(u)$ denote the set of all pendant vertices which are adjacent to $u$. For $1 \leq i \leq s-1$,

let us define $R\left(u_{i}\right)=\left\{u_{i}\right\} \cup P\left(u_{i+1}\right)$

$A=\cup_{i} R\left(u_{i}\right)$, for $1 \leq i \leq s, i-o d d$,

and $B=\left(\bigcup_{i} R\left(u_{i}\right)\right) \cup P\left(u_{1}\right)$, for $2 \leq i \leq s, i-$ even 
From the above, clearly $(A, B)$ defines a bipartition of $T_{1}$. Now label the vertices of $A$, that is, $a_{1}, a_{2}, \ldots, a_{p}$ by $m, m-1, m-2$, $\ldots, m-(p-1)$ and the vertices of $B$, that is, $b_{1}, b_{2}, \ldots, b_{q}$ by $0,1,2,3, \ldots, q-1$. Further observe that the edges values to be realized that are in transition between the copies are realized with the unique incident to the penultimate vertex. Clearly all the edge values of are distinct and varies from $1,2,3, \ldots, m$ and hence the above labeling gives the required graceful labeling of $T_{1}$.

The illustration corresponding to the proof of the theorem 2.1 is provided through the figures $1,2,3$ in appendix.

Now let us proceed to our main result.

For $i \geq 2$, consider the tree $T_{i-1}$ having $k$ attachment vertices and by induction assume that $T_{i-1}$ is graceful.

Let $S_{i-1}$ be any caterpillar not necessarily the same taken before. Let $S_{i-1}^{1}, S_{i-1}^{2}, S_{i-1}^{3}, \ldots, S_{i-1}^{t}$ be the copies of $S_{i-1}$. Consider the tree $T_{i-1}$ without graceful numbering. Now construct the tree $T_{i}\left(=T_{i-1}+S_{i-1}\right)$ by merging the penultimate vertex of each copy of $S_{i-1}^{j}$ for $1 \leq j \leq t$ with every attachment vertex of $T_{i-1}$.

Theorem 2.2: The tree $T_{i}$ is graceful for $i \geq 2$.

Proof: Consider the graph $T_{i}$ with $N$ edges. As same like theorem 2.1 , for $1 \leq i \leq t$ name the unlabelled attachment vertices of each copy $S_{i-1}$ and let it be $u_{1}, u_{2}, u_{3}, \ldots u_{M}$.

For $1 \leq i \leq M-1$,

let us define $R\left(u_{i}\right)=\left\{u_{i}\right\} \cup P\left(u_{i+1}\right)$

$A=\bigcup_{i} R\left(u_{i}\right)$, for $1 \leq i \leq M, i-o d d$

and $B=\left(\cup_{i} R\left(u_{i}\right)\right) \cup P\left(u_{1}\right)$, for $2 \leq i \leq M, i-$ even

From the above, clearly $(A, B)$ defines a bipartition of $T_{i}$. Now label the vertices of $A$, that is, $a_{1}, a_{2}, \ldots, a_{p}$ by $N, N-1, N-2$, $\ldots, N-(p-1)$ and the vertices of $B$, that is, $b_{1}, b_{2}, \ldots, b_{q}$ by $0,1,2,3, \ldots, q-1$. Clearly all the edge values are distinct and varies from $1,2,3, \ldots, N$. Thus $T_{i}$ is graceful.

\section{Conclusion}

It is noted that for $i \geq 1$, the tree $T_{i}$ is constructed recursively from $T_{i-1}$ by attaching a caterpillar $S_{i-1}$. Thus if $S_{i-1}$ is chosen to be any tree, not necessarily a caterpillar, whether still $T_{i}$ admits graceful labeling.

Conjecture 1. The tree $T_{i}=T_{i-1}+S_{i-1}$ is graceful for $i \geq 1$ where $S_{i-1}$ is any graceful tree.

As $T_{0}$ is graceful, the tree $T_{i}$ generated by this attachment process also resulted the graceful labeling, thus by choosing $T_{0}$ as any graceful tree, whether $T_{i}$ admits graceful labeling.

Conjecture 2. The tree $T_{i}=T_{i-1}+S_{i-1}$ is graceful for $i \geq 1$ where $T_{0}$ is any graceful tree.

\section{Acknowledgement}

The authors thankfully acknowledge the referee for their valuable comments in improving the presentation of the paper. Further the author thank the management of SASTRA University for providing support in publishing this paper.

\section{References}

[1] Golomb.S.W, How to number a graph in graph theory and computing. In: Read R.C. (ed.) Academic Press, New York, (1972), 23-37.

[2] Rosa.A, On certain valuations of the vertices of a graph, Theory of Graphs International Symposium, Rome, July) Gordon and Breach, N.Y. and Dunod Paris, (1966), 105-110.

[3] Stanton.R and Zarnke.C, Labeling of balanced trees, Proc. 4th Southeast Conf.Combin., Graph Theory, Comput., (1973), 479-495.

[4] Bahl. P, Lake.S and Wertheim.A, Gracefulness of families of spiders, Involve 3 (2010), 241-247.

[5] Burzio.M and Ferraeese.G, The subdivision of a graceful tree is a graceful tree, Discrete Math, 181 (1988), 275-281.

[6] Chen.W.C, Lu.H.I. and Yeh, Operations of interlaced trees and graceful trees, Southeast Asian Bull. Math., 21 (1997), 337-348.

[7] Gallian.J.A, A dynamic survey of Graph labeling, The Electronic Journal of Combinatorics,, DS6 (2017).

[8] Koh.K.M, Rogers.D.G..and Tan. T, Product of graceful trees, Discrete Math., 31 (1980), 2790-292.

[9] Poljak.S and Sura.M, An algorithm for graceful labeling of a class of symmetrical trees, Ars Combin., 14 (1982), 57-66.

[10] Sethuraman.G and Jesintha.J, All extended banana trees are graceful, Proc.Internat. Conf. Math. Comput. Sci., 1 (2009), 4-8.

[11] Sethuraman.G and J. Jesintha, All banana trees are graceful, Advances Appl. Disc. Math.,, 4 (2009), 53-64.

[12] Sethuraman.G and J.Jesintha.J, A new class of graceful lobsters, J. Combin. Math. Combin. Computing, 67 (2008), 99-109.

[13] Sethuraman.G and P. Ragukumar.J, Every Tree is a subtree of Graceful Tree, Graceful Graph and Alpha-labeled Graph, Ars Comb., 132 (2017), 27-47.

[14] Sethuraman.G and Venkatesh. S, Decomposition of complete graphs and complete bipartite graphs into alpha labelled trees, Ars Combinatoria, 93 (2009), 371-385.

[15] S. Venkatesh, Bharathi.S, On generating Graceful trees, International Journal of Pure and Applied Mathematics, 118(9), 2018, 899. 904.

\section{APPENDIX}

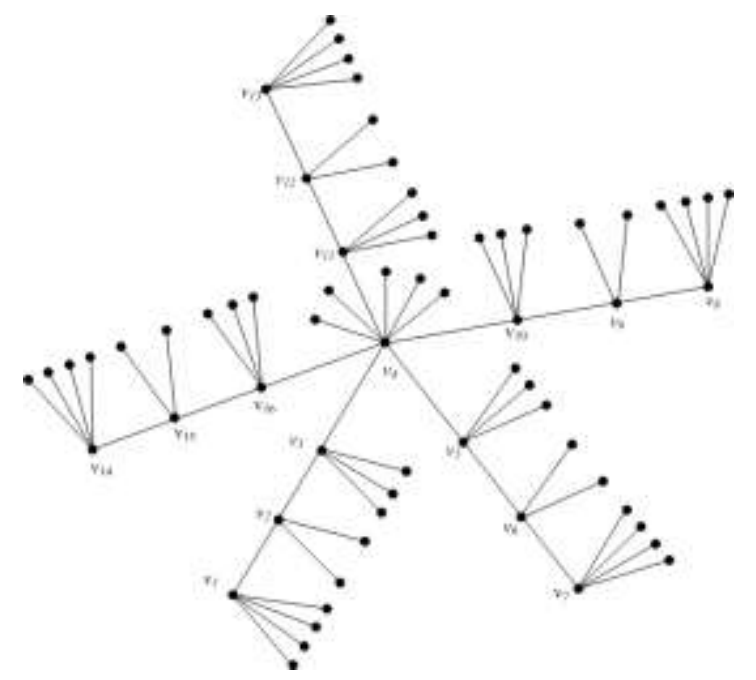

Fig.1:. The tree $T_{0}$ with the attachment vertices

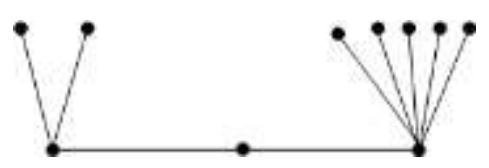

Fig.2:. The tree $S_{0}$ 


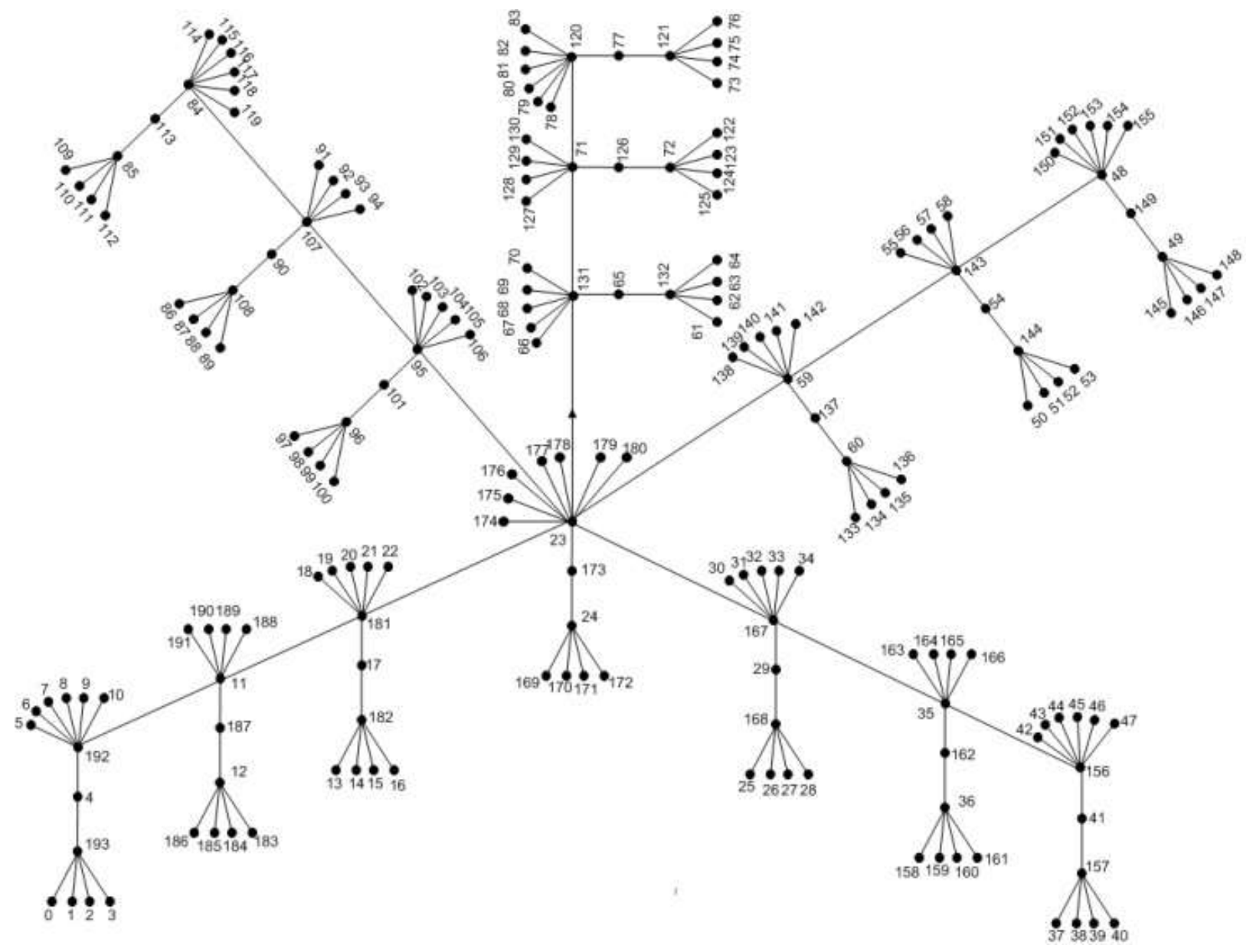

Fig.3: The tree $T_{1}=T_{0}+S_{0}$ 